\title{
The relationship between the number of natural teeth and airflow obstruction: a cross-sectional study using data from the Korean National Health and Nutrition Examination Survey
}

\author{
This article was published in the following Dove Press journal: \\ International Journal of COPD \\ 21 December 2015 \\ Number of times this article has been viewed
}

\author{
Sei Won Kim ${ }^{1}$ \\ Kyungdo $\mathrm{Han}^{2}$ \\ Shin Young $\mathrm{Kim}^{3}$ \\ Chan Kwon Park ${ }^{3}$ \\ Chin Kook Rhee' \\ Hyoung Kyu Yoon ${ }^{3}$ \\ 'Department of Internal Medicine, \\ Seoul St Mary's Hospital, College of \\ Medicine, The Catholic University \\ of Korea, Seoul, Republic of Korea, \\ ${ }^{2}$ Department of Medical Statistics, \\ College of Medicine, The Catholic \\ University of Korea, Seoul, Republic \\ of Korea, ${ }^{3}$ Department of Internal \\ Medicine, Yeouido St Mary's Hospital, \\ College of Medicine, The Catholic \\ University of Korea, Seoul, Republic \\ of Korea
}

Correspondence: Hyoung Kyu Yoon Department of Internal Medicine, Yeouido St Mary's Hospital, College of Medicine, The Catholic University of Korea, 62 Yeouido-dong,

Yeongdeungpo-gu, Seoul I50-7|3,

Republic of Korea

Tel +82 23779 II I 3

Email cmcyhg@catholic.ac.kr
Background: Potential associations between oral health and respiratory disease, including chronic obstructive pulmonary disease (COPD), have been suggested in several studies. Among the indicators reflecting oral health, the number of natural teeth is an integrated and simple index to assess in the clinic. In this study, we examined the relationship between the number of natural teeth and airflow obstruction, which is a central feature of COPD.

Methods: A total of 3,089 participants over 40 years, who underwent reliable spirometry and oral health assessments were selected from the Korean National Health and Nutrition Examination Survey 2012, a cross-sectional and nationally representative survey. Spirometry results were classified as normal, restrictive, or obstructive pattern. Total number and pairs of natural teeth were counted after excluding third molars.

Results: After adjusting for other variables, such as age, body mass index, socioeconomic factors, and oral health factors, the group with airflow obstruction showed significantly fewer natural teeth than the other groups in males $(P=0.014$ and 0.008 for total number and total pairs of natural teeth, respectively). Compared with participants with full dentition, the adjusted odds ratio for airflow obstruction in males with fewer than 20 natural teeth was 4.18 (95\% confidence interval: 2.06-8.49) and with fewer than 10 pairs of natural teeth was 4.74 (95\% confidence interval: 2.34-9.62). However, there was no significant association between the total number or pairs of natural teeth and airflow obstruction after adjustment in females.

Conclusions: Loss of natural teeth was significantly associated with the presence of airflow obstruction in males. Our finding suggests that the number of natural teeth could be one of the available indices for obstructive lung diseases, including COPD.

Keywords: tooth loss, oral health, airflow obstruction, chronic obstructive pulmonary disease

\section{Introduction}

Chronic obstructive pulmonary disease (COPD), a lung disease characterized by airflow limitations that are not fully reversible, is a major cause of chronic morbidity and mortality worldwide. ${ }^{1-3}$ The Global Burden of Disease study predicted that COPD is predicted to rise from the sixth most common cause of mortality worldwide, to the third most common cause of mortality in $2020 .{ }^{4}$ Additionally, with the aging of the population, the burden of COPD is increasing continuously. ${ }^{5-7}$ However, recent data likely underestimate the total burden of COPD because the disease is usually not diagnosed until it is clinically apparent and already moderately advanced. ${ }^{8}$

Although COPD is primarily a pulmonary disease associated with an abnormal inflammatory response in the lungs, COPD is also associated with a certain degree 
of systemic inflammation, which has been correlated with adverse clinical effects. ${ }^{9-11}$ Several studies have reported potential associations between oral health and respiratory diseases, including COPD. ${ }^{12-14}$ Scannapieco and Mylotte ${ }^{15}$ suggested that periodontal disease might change oral microbiological conditions and permit mucosal colonization and infection by respiratory pathogens. ${ }^{15}$ Additionally, oral health is likely to interact with other cofactors (eg, smoking, environmental pollutants, infection, allergy, genetic factors) that are also important in COPD patients. ${ }^{16}$ Among indicators of oral health, the number of natural teeth can be rapidly and easily determined in the clinic. Öztekin et $\mathrm{al}^{17}$ reported that the number of teeth was significantly lower in COPD patients. Barros et $\mathrm{al}^{11}$ reported that edentulous COPD patients were at greater risk of having a COPD-related event (hospitalization and death).

The aim of this study was to investigate the association between the number of natural teeth and airflow obstruction, which is a central feature of COPD, by analyzing data from the Korean population.

\section{Materials and methods Study population}

Data were collected from the Korean National Health and Nutrition Examination Survey (KNHANES), which was performed by the Korean Centers for Disease Control and Prevention (Korean Ministry of Health and Welfare) in 2012. ${ }^{18}$ KNHANES has been conducted regularly since 1998 to monitor the general health and nutritional status of the civilian, noninstitutionalized South Korean population. Participants were selected using proportional allocation-systemic sampling with multistage stratification to produce a nationally representative sample of the resident, civilian Korean population. Prior to the national survey, all participants provided written informed consent. Moreover, all databases were anonymized. KNHANES comprised three sections: a health interview survey, a health examination, and a nutrition survey. ${ }^{18}$ Additional details about the KNHANES have been provided elsewhere. ${ }^{18}$ Among participants in the survey in 2012, this current study analyzed participants over 40 years who underwent reliable spirometry and oral health assessments $(n=3,089 ; 1,291$ males and 1,798 females). The age range was $40-88$ years for males and $40-87$ years for females. Participants with asthma were excluded according to medical history. This current study was approved by the Institutional Review Board of Seoul St Mary's Hospital. The Institutional Review Board of Seoul St Mary's Hospital did not require patient consent for this present study because all participants gave their consent for the KNHANES before the registration, and the KNHANES data is open to the public after being anonymized.

\section{Measurements and variables}

Height, weight, and waist circumference (WC) were measured with standard procedures. Height was measured to the nearest $0.1 \mathrm{~cm}$. WC was measured at the midpoint of the lower margin of the 12th rib and the iliac crest in the midaxillary line at the end of expiration. Weight was measured to the nearest $0.1 \mathrm{~kg}$ and body mass index (BMI) was calculated as weight in kilograms divided by height in meters squared.

All participants were asked about their demographic characteristics, socioeconomic characteristics, and medical history. Trained interviewers performed the interviews using structured questionnaires. Smoking status was categorized as ever smokers and nonsmokers. Subjects were considered as ever smokers if they had smoked $>100$ cigarettes in their lifetime. The amount of alcohol consumed (in g/day) was calculated using the average number of alcoholic beverages ingested and frequency of drinking. Subjects who drank more than $30 \mathrm{~g}$ /day were deemed heavy drinkers. Monthly income was standardized according to the number of family members (monthly income/ $\sqrt{\text { number of family }}$ members) and was divided into four quartile groups: lowest, lower middle, higher middle, and highest (Table S1). Low income corresponded to the lowest quartile of household income. Education level was classified as high, if the subjects finished high school education or more (higher than 12th grade). Geographic regions were classified as rural (Gangwon, Chungbuk, Chungnam, Jeonbuk, Jeonnam, Gyeongbuk, Gyeongnam, and Jeju) or urban (Seoul, Busan, Daegu, Daejeon, Gwangju, Incheon, Ulsan, and Gyeonggi) areas. Marriage was checked from the presence of a spouse. Regular exercise was defined as strenuous physical activity performed for at least 20 minutes at a time at least three times per week.

The nutrition surveys included questions about the participant's eating pattern, use of dietary supplements, knowledge of nutrition, and food intake using the 24 hours recall method. The total energy intake and the percentage of energy from each nutrient (fat, carbohydrate, and protein) were then calculated.

Diabetes mellitus (DM) was defined as a fasting glucose level $\geq 126 \mathrm{mg} / \mathrm{dL}$, the current use of antidiabetic medications, or a self-reported physician diagnosis of DM. Metabolic syndrome was defined using the diagnostic guidelines from the American Heart Association and the National Heart, Lung, and Blood Institute. ${ }^{19}$ Vitamin D is well known for its crucial role in bone homeostasis. Vitamin D showed significant association with pulmonary function, periodontitis, and tooth loss as seen from other studies. ${ }^{20,21}$ Vitamin D level 
was measured with a 1470 Wizard $\gamma$-counter (Perkin-Elmer, Turku, Finland) using a radioimmunoassay.

\section{Spirometry and definition of airflow obstruction}

Licensed trained technicians measured the forced vital capacity (FVC), forced expiratory volume in 1 second $\left(\mathrm{FEV}_{1}\right)$, and the $\mathrm{FEV}_{1} / \mathrm{FVC}$ ratio using a dry rolling seal spirometer (model 2130; SensorMedics, Yorba Linda, CA, USA). A quality-control program was performed; the spirometer was calibrated every morning before performing spirometry (and recalibrated every time the room temperature changed by $3^{\circ} \mathrm{C}$ ), and the American Thoracic Society/European Respiratory Society criteria of acceptability and repeatability were followed by the examiners on site. All of the spirometry values were described as prebronchodilator results. Normal predicted values were derived from large population studies of healthy subjects. Variables used to predict normal values included age, sex, height, and ethnicity. ${ }^{22}$ Spirometry results were classified as normal, restrictive, and obstructive pattern. Subjects with $\mathrm{FEV}_{1} / \mathrm{FVC} \geq 70 \%$ and $\mathrm{FVC} \geq 80 \%$ of the normal predicted value were considered normal. ${ }^{23,24}$ When $\mathrm{FEV}_{1} / \mathrm{FVC} \geq 70 \%$ and $\mathrm{FVC}$ was under $80 \%$ of the normal predicted value, the subjects were classified as restrictive pattern. Obstructive pattern, which we considered to have airflow obstruction in this study, was defined with $\mathrm{FEV}_{1} / \mathrm{FVC}<70 \%$. The severity of airflow obstruction was based on the percentage of normal predicted prebronchodilator $\mathrm{FEV}_{1}$.

\section{Number and pairs of natural teeth}

The KNHANES 2012 oral health data recorded the status for each of the 32 teeth. In this study, total number and pairs of natural teeth were counted after excluding third molars. Based on this information, we categorized the subjects into three groups according to total numbers of natural teeth: full dentition (28 teeth), 20-27 teeth, and fewer than 20 teeth. We also classified the subjects into one of three groups according to pairs of natural teeth.

\section{Other oral health assessment}

The World Health Organization community periodontal index (CPI) was used to assess periodontitis and when CPI $\geq$ code 3 , we deemed this to be periodontitis. ${ }^{25,26}$ Code 3 indicates that at least one site had a $>3.5 \mathrm{~mm}$ pocket in the index teeth, which are $2,3,8,14,15,18,19,24,30$, and 31 , according to the Universal Numbering System, which has been adopted by the American Dental Association. A CPI probe, which is appropriate for the World Health Organization guidelines, was used. We also checked the number of tooth brushings and the use of secondary oral products for assessing oral health behavior. Number of tooth brushings was categorized as once, twice, and three or more times per day. Secondary oral products included mouthwash, dental floss, water flosser, an interdental brush, and an electric toothbrush.

\section{Statistical analysis}

All data were presented as mean \pm standard error for continuous variables or as proportions (standard error) for categorical variables. Logarithmic transformation was used to analyze variables with skewed distributions. Statistical analyses were performed with the SAS survey procedure (ver 9.3; SAS Institute, Inc., Cary, NC, USA) to reflect the complex sampling design and sampling weights of KNHANES and to provide nationally representative prevalence estimates. The $\chi^{2}$ test was used with categorical variables and a one-way analysis of variance with continuous variables. We used analysis of covariance to compare the number and pairs of the natural teeth according to lung function after adjusting for potential confounders. Multivariate logistic regression analyses were performed to estimate the association of airflow obstruction and the number of natural teeth. Odds ratio (OR) and 95\% confidence intervals (CIs) were estimated after adjustment for potential confounders. In the multivariate analyses for the number and pairs of natural teeth, we first adjusted for age (model 1) and then adjusted for the variables in model 1 plus smoking, drinking, exercise, income, education, and BMI (model 2). Finally, model 3 adjusted for the variables in model 2 plus periodontitis, number of tooth brushings, and the use of secondary oral products. $P$-values $<0.05$ were considered to indicate statistical significance.

\section{Results}

Baseline characteristics of study population are shown in Table 1. All subjects were categorized into three groups (normal, restrictive, and obstructive) according to their pulmonary function test. Among the total population, 12.9\% had obstructive lung function, $10.0 \%$ had restrictive lung function, and $77.2 \%$ had normal lung function. Difference between males and females was $20.1 \%$ versus $6.0 \%, 8.9 \%$ versus $11.0 \%$, and $71.0 \%$ versus $83.0 \%$ for obstructive, restrictive, and normal lung function, respectively. There were significant differences among the three groups in age $(P<0.001$, both), height $(P<0.001$ and $P=0.017$, respectively $)$, weight $(P<0.001$, both), BMI $(P<0.001$, both $)$, and WC $(P<0.001$ and $P=0.002$, respectively) in males and females. The obstructive group was older and had lower weight and BMI. The restrictive group showed higher weight, BMI, and WC. The normal group was taller than the other groups. 
Table I Baseline characteristics of the participants

\begin{tabular}{|c|c|c|c|c|c|c|c|c|}
\hline \multirow[t]{2}{*}{ Variables } & \multicolumn{3}{|l|}{ Male groups } & \multirow[t]{2}{*}{$P$-value } & \multicolumn{3}{|c|}{ Female groups } & \multirow[t]{2}{*}{$P$-value } \\
\hline & Normal & Restrictive & Obstructive & & Normal & Restrictive & Obstructive & \\
\hline Age (year) & $51.4 \pm 0.4$ & $55.8 \pm 1$ & $61.9 \pm 0.7$ & $<0.00$ I & $54.5 \pm 0.4$ & $57.2 \pm 0.9$ & $63.9 \pm 1.4$ & $<0.001$ \\
\hline Height (cm) & $169.6 \pm 0.3$ & $167.3 \pm 0.7$ & $167.5 \pm 0.4$ & $<\mathbf{0 . 0 0 I}$ & $155.9 \pm 0.2$ & $154.8 \pm 0.6$ & $153.6 \pm 0.9$ & 0.017 \\
\hline Weight (kg) & $70.4 \pm 0.4$ & $74.2 \pm 1.1$ & $66.8 \pm 0.7$ & $<0.001$ & $59 \pm 0.3$ & $60.5 \pm 0.9$ & $54.8 \pm 0.9$ & $<0.001$ \\
\hline BMI $\left(\mathrm{kg} / \mathrm{m}^{2}\right)$ & $24.4 \pm 0.1$ & $26.5 \pm 0.3$ & $23.8 \pm 0.2$ & $<0.001$ & $24.3 \pm 0.1$ & $25.2 \pm 0.4$ & $23.2 \pm 0.4$ & $<0.001$ \\
\hline WC $(\mathrm{cm})$ & $85.1 \pm 0.3$ & $90.4 \pm 0.9$ & $85.5 \pm 0.5$ & $<0.001$ & $80.7 \pm 0.4$ & $83.9 \pm 0.9$ & $80.1 \pm I . I$ & 0.002 \\
\hline Ever smokers (\%) & $80.8(1.7)$ & $78.3(3.6)$ & $87.3(2.2)$ & 0.040 & $8.3(1.1)$ & $5.8(1.9)$ & $12.2(4.1)$ & 0.261 \\
\hline Heavy drinker (\%) & $37(2)$ & $37.1(5.9)$ & $38.7(3.6)$ & 0.929 & $3.9(0.7)$ & $3(1.8)$ & $3.4(3.3)$ & 0.924 \\
\hline Income (lowest quartile, \%) & $9.8(1.3)$ & $11.5(3.5)$ & $20.8(2.7)$ & $<0.001$ & $18(1.4)$ & $26.4(4.5)$ & $38.1(7.2)$ & $<0.001$ \\
\hline Education ( $>12$ years, \%) & $86.5(1.5)$ & $82.8(3.6)$ & $68.9(3.6)$ & $<\mathbf{0 . 0 0 1}$ & $68.1(1.6)$ & $64.7(4.5)$ & $34.7(5.9)$ & $<0.001$ \\
\hline Living place (rural, \%) & $19.6(3.4)$ & $27.3(7)$ & $25.5(5)$ & 0.212 & $19.6(3.1)$ & I8.3 (4.6) & $27.5(8)$ & 0.352 \\
\hline Marriage (\%) & $92.2(1.1)$ & $92.3(3.2)$ & $89.1(2.2)$ & 0.417 & $81.4(1.2)$ & $82.4(3.3)$ & $62.1(6.1)$ & $<0.001$ \\
\hline Exercise (\%) & $20.8(1.7)$ & $15.8(3.4)$ & $14(2.3)$ & 0.042 & $13.9(1.2)$ & $10.9(2.1)$ & II .4 (3.9) & 0.506 \\
\hline Total energy (kcal/day) & $2,382.4 \pm 42.7$ & $2,234.3 \pm 109.7$ & $2,188.4 \pm 67.3$ & $0.04 I$ & 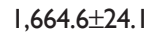 & 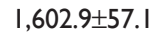 & I,529.9 \pm 51.9 & 0.054 \\
\hline $\mathrm{DM}(\%)$ & $10.1(1.1)$ & $19.3(3.8)$ & I6.I (2.6) & 0.003 & $9(0.9)$ & I7.I (3.8) & $15.4(3.4)$ & 0.002 \\
\hline Metabolic syndrome (\%) & $32.9(2)$ & $58.8(5.2)$ & $32.9(3.2)$ & $<0.001$ & $37.9(1.9)$ & $57.2(4.3)$ & $42.1(5.7)$ & $<0.001$ \\
\hline Vitamin D (ng/mL) & $18.6 \pm 0.3$ & $17.4 \pm 0.6$ & $19.7 \pm 0.5$ & 0.005 & $16.6 \pm 0.2$ & $16.3 \pm 0.5$ & $17 . \mid \pm 0.7$ & 0.616 \\
\hline Number of natural teeth & $25.4 \pm 0.2$ & $24.6 \pm 0.4$ & $21.8 \pm 0.5$ & $<0.001$ & $24.4 \pm 0.2$ & $23.7 \pm 0.4$ & $21.4 \pm 0.7$ & $<0.001$ \\
\hline Periodontitis (\%) & $38.1(2.5)$ & $4 \mathrm{I}(5.7)$ & $44.1(4)$ & 0.359 & $25.4(1.7)$ & $26.5(4.3)$ & $26.7(4.5)$ & 0.934 \\
\hline Number of tooth brushings & & & & 0.490 & & & & 0.102 \\
\hline I/day (\%) & I4.8 (I.5) & I7.5 (4.4) & $17.3(2.5)$ & & $8.9(0.9)$ & $10.9(3.7)$ & $14.4(3.6)$ & \\
\hline 2/day (\%) & $42.2(2.1)$ & $33.3(4.8)$ & $42.1(3.3)$ & & $41.2(1.7)$ & $48.7(5.1)$ & $48.5(5.2)$ & \\
\hline$\geq 3 /$ day $(\%)$ & $43(2.3)$ & $49.2(5.4)$ & $40.7(3.5)$ & & $49.9(1.6)$ & $40.4(4.8)$ & $37.1(5.3)$ & \\
\hline Secondary oral products (\%) & $45.9(2.3)$ & $42.7(4.9)$ & $37.5(3.4)$ & 0.106 & $50.2(1.6)$ & $49(5.2)$ & $43.4(6.3)$ & 0.588 \\
\hline
\end{tabular}

Notes: Data represent the mean \pm standard error or percentages (standard error). The statistically significant values are shown in bold. Abbreviations: BMI, body mass index; WC, waist circumference; DM, diabetes mellitus.

In socioeconomic characteristics, there were significant differences in income $(P<0.001$, both $)$ and education $(P<0.001$, both $)$ in males and females. The obstructive group showed lower income and education than the other groups. In males, the obstructive group showed significantly more smokers $(P=0.040)$, less exercise $(P=0.042)$, and total energy $(P=0.041)$. There were more unmarried females in the obstructive group $(P<0.001)$. Although the amount of total energy was smaller in the obstructive group in females, the difference was of borderline significance $(P=0.054)$.

DM $(P=0.003$ and $P=0.002)$ and metabolic syndrome $(P<0.001$, both $)$ were significantly more frequent in the restrictive group (males and females). Vitamin D levels were significantly higher in the obstructive group in males ( $P=0.005)$.

In terms of oral health, number of natural teeth was significantly lower in the obstructive group in males and females $(P<0.001$, both). Periodontitis index, number of tooth brushings, and use of secondary oral products showed no significant difference in the three groups. In both males and females, the proportion of participants with full dentition and all pairs of natural teeth tended to decrease in the order normal, restrictive, and obstructive group (Figure 1A and B). The obstructive group showed considerable decreases versus the other groups. The proportion of individuals who had fewer than 20 natural teeth or fewer than 10 pairs of natural teeth showed a reverse pattern, in both males and females. Number and pairs of natural teeth showed the same pattern and distribution. However, males showed greater changes in the trend and distribution than females.

We adjusted for variables that might influence the association between total number of natural teeth and lung function (Table 2). After adjusting for age, BMI, socioeconomic status (smoking, drinking, exercise, income, education), and oral health indexes (periodontitis, number of tooth brushings, and use of secondary oral products), the obstructive group showed significantly fewer natural teeth than the other groups in males ( $P=0.014$ and $P=0.008$ for total number and total pairs of natural teeth, respectively). There was no significant difference after adjustment in females in the total number or pairs of natural teeth.

The subjects were divided into three groups according to the total number $(<20,20-27,28)$ and pairs $(<10,10-13$, 14) of natural teeth (Table 3). After adjusting for age, BMI, socioeconomic status, and oral health indexes, ORs for 

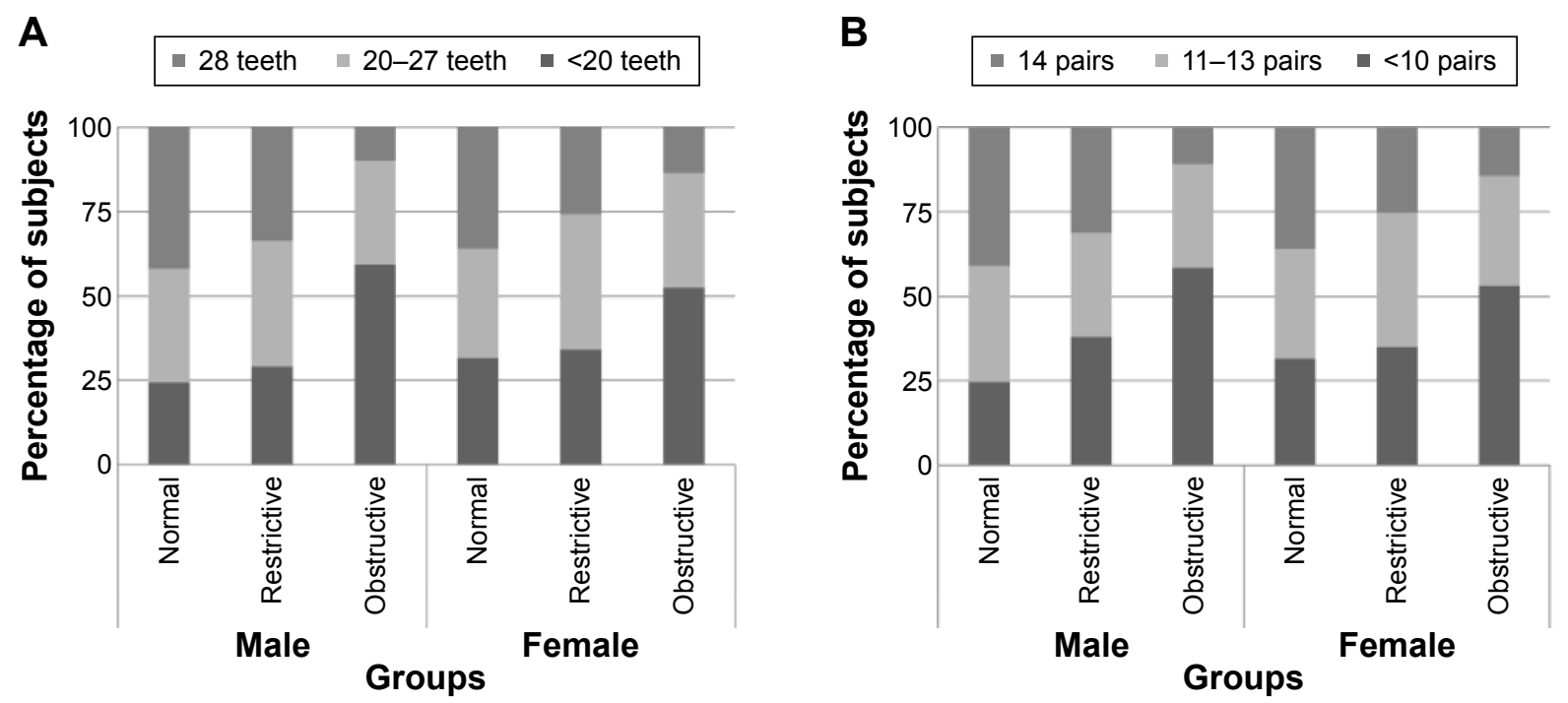

Figure I Distribution of number of natural teeth $(\mathbf{A})$ and pairs of natural teeth $(\mathbf{B})$ with lung function.

Notes: In both males and females, the proportion of participants with full dentition and all pairs of natural teeth tended to decrease relative to the order of normal, restrictive, and obstructive group. The obstructive group showed considerable decreases versus the other groups. The proportion of individuals who had fewer than 20 natural teeth or fewer than 10 pairs of natural teeth showed a reverse pattern, in both males and females. Number and pairs of natural teeth showed the same pattern and distribution. However, males showed greater changes in the trend and distribution than females.

airflow obstruction were $4.18(95 \% \mathrm{CI}=2.06-8.49)$ and $3.21(95 \% \mathrm{CI}=1.85-5.58)$ in the groups with total numbers of teeth below 20 and 20-27, respectively. The adjusted OR for airflow obstruction of total pairs of natural teeth was 4.74 (95\% CI $=2.34-9.62)$ and 3.08 (95\% CI $=1.79-5.32)$ in the groups with total pairs of teeth below 10 and 10-13, respectively. There was no significant relationship between the number or pairs of natural teeth and airflow obstruction in females.

Figure 2 shows the trend of $\mathrm{FEV}_{1}$ with subgroups of the number of natural teeth in patients with airflow obstruction. In both sexes, $\mathrm{FEV}_{1}$ showed a decreasing trend in the groups with 20-27 teeth and $<20$ teeth compared with the 28 -teeth group after adjustment for confounding factors (age, smoking, drinking, exercise, income, education, and BMI).

\section{Discussion}

Although our study is a cross-sectional study using the results of prebronchodilator spirometry, we detected a statistically significant association between the number or pairs of natural teeth and airflow obstruction in males, even after adjusting for other variables such as age, BMI, socioeconomic factors (smoking, drinking, exercise, income, education), and oral health factors (periodontitis, number of tooth brushings, use of secondary oral products). The adjusted OR for airflow obstruction in males with fewer than 20 natural teeth was $4.18(2.06-8.49)$ and that in males with fewer than 10 pairs of natural teeth was 4.74 (2.34-9.62). However, there was no significant association between the number or pairs of natural teeth and airflow obstruction in females. Within patients with airflow obstruction, percentage of normal predicted FEV

Table 2 Multivariate adjusted number and pairs of natural teeth and lung function

\begin{tabular}{|c|c|c|c|c|c|c|c|c|}
\hline & \multicolumn{3}{|c|}{ Male groups } & \multirow[t]{2}{*}{$P$-value } & \multicolumn{3}{|c|}{ Female groups } & \multirow[t]{2}{*}{$P$-value } \\
\hline & Normal & Restrictive & Obstructive & & Normal & Restrictive & Obstructive & \\
\hline \multicolumn{9}{|c|}{ Total number of natural teeth } \\
\hline Model Ia & $24.0 \pm 0.2$ & $24.1 \pm 0.4$ & $22.6 \pm 0.4$ & 0.012 & $23.4 \pm 0.2$ & $23.5 \pm 0.3$ & $23.1 \pm 0.6$ & 0.806 \\
\hline Model $2^{b}$ & $23.9 \pm 0.2$ & $24.4 \pm 0.4$ & $22.7 \pm 0.4$ & 0.015 & $23.5 \pm 0.2$ & $23.7 \pm 0.3$ & $23.2 \pm 0.6$ & 0.752 \\
\hline Model $3^{c}$ & $23.9 \pm 0.2$ & $24.1 \pm 0.3$ & $22.8 \pm 0.4$ & 0.014 & $23.4 \pm 0.1$ & $23.6 \pm 0.3$ & $23.5 \pm 0.6$ & 0.798 \\
\hline \multicolumn{9}{|c|}{ Total pairs of natural teeth } \\
\hline Model I ${ }^{\mathrm{a}}$ & $10.8 \pm 0.2$ & $10.8 \pm 0.3$ & $9.8 \pm 0.3$ & 0.005 & $10.4 \pm 0.1$ & $10.5 \pm 0.2$ & $10.3 \pm 0.4$ & 0.876 \\
\hline Model $2^{\mathrm{b}}$ & $10.8 \pm 0.2$ & $10.9 \pm 0.3$ & $9.9 \pm 0.3$ & 0.012 & $10.5 \pm 0.1$ & $10.6 \pm 0.2$ & $10.4 \pm 0.4$ & 0.801 \\
\hline Model $3^{c}$ & $10.8 \pm 0.1$ & $10.7 \pm 0.2$ & $9.9 \pm 0.2$ & 0.008 & $10.5 \pm 0.1$ & $10.6 \pm 0.2$ & $10.6 \pm 0.4$ & 0.836 \\
\hline
\end{tabular}

Notes: Data represent the mean \pm standard error. The statistically significant values are shown in bold. Adjusted for age; bvariables in model I plus smoking, drinking, exercise, income, education, and BMI; 'variables in model 2 plus periodontitis, number of tooth brushings per day, and use of secondary oral products. Abbreviation: BMI, body mass index. 
Table 3 Risk of airflow obstruction according to the number or pairs of natural teeth

\begin{tabular}{|c|c|c|c|c|c|c|}
\hline & Male & & & Female & & \\
\hline Total number of natural teeth & $<20$ teeth & 20-27 teeth & 28 teeth & $<20$ teeth & 20-27 teeth & 28 teeth \\
\hline Model Ia & $3.12(1.62-6.01)$ & $2.47(1.54-3.94)$ & I (Ref) & I.0I $(0.46-2.20)$ & $1.60(0.78-3.27)$ & I (Ref) \\
\hline Model $2^{b}$ & $2.80(1.44-5.44)$ & $2.40(1.48-3.89)$ & I (Ref) & $0.94(0.40-2.24)$ & $1.54(0.77-3.06)$ & I (Ref) \\
\hline Model 3c & $4.18(2.06-8.49)$ & $3.21(1.85-5.58)$ & I (Ref) & $0.54(0.17-1.73)$ & $0.99(0.37-2.65)$ & I (Ref) \\
\hline Total pairs of natural teeth & $<10$ pairs & $10-13$ pairs & 14 pairs & $<10$ pairs & 10-13 pairs & 14 pairs \\
\hline Model Ia & $3.33(1.82-6.05)$ & $2.28(1.44-3.62)$ & I (Ref) & I. $17(0.56-2.47)$ & $1.65(0.80-3.39)$ & I (Ref) \\
\hline Model $2^{b}$ & 3.00 (I.6I-5.59) & $2.24(1.40-3.60)$ & I (Ref) & $1.14(0.55-2.35)$ & $1.58(0.78-3.18)$ & I (Ref) \\
\hline Model $3^{c}$ & 4.74 (2.34-9.62) & 3.08 (1.79-5.32) & I (Ref) & $0.66(0.23-1.93)$ & $1.03(0.38-2.77)$ & I (Ref) \\
\hline
\end{tabular}

Notes: Results represent odds ratio ( $95 \%$ confidence interval). The statistically significant values are shown in bold. Adjusted for age; bvariables in model I plus smoking, drinking, exercise, income, education, and BMl; 'variables in model 2 plus periodontitis, number of tooth brushings per day, and use of secondary oral products.

Abbreviation: BMI, body mass index.

tended to decrease with tooth loss. However, there was no statistically significant relationship between the number of natural teeth and percentage of normal predicted $\mathrm{FEV}_{1}$.

Previous studies have suggested an association between airflow obstruction, which is a central feature of COPD, and poor oral health. ${ }^{12,16,27-30}$ Scannapieco et al ${ }^{12}$ investigated the association between oral conditions and respiratory disease using data from the National Health and Nutrition Examination Survey I. ${ }^{12}$ Subjects with maximum oral hygiene index values were 4.5 -fold more likely to have a chronic respiratory disease than those with an oral hygiene index of 0 in their study. Hayes et $\mathrm{al}^{28}$ investigated the association between COPD and periodontal disease, assessed by radiographic alveolar bone loss. They showed that increased alveolar bone loss was associated with an increased risk of COPD. A case-control study with 581 COPD cases and 438 non-COPD controls was performed by $\mathrm{Si}$ et $\mathrm{al}^{27}$ in a Chinese population. The results showed a strong association between periodontitis and COPD, and plaque index was a

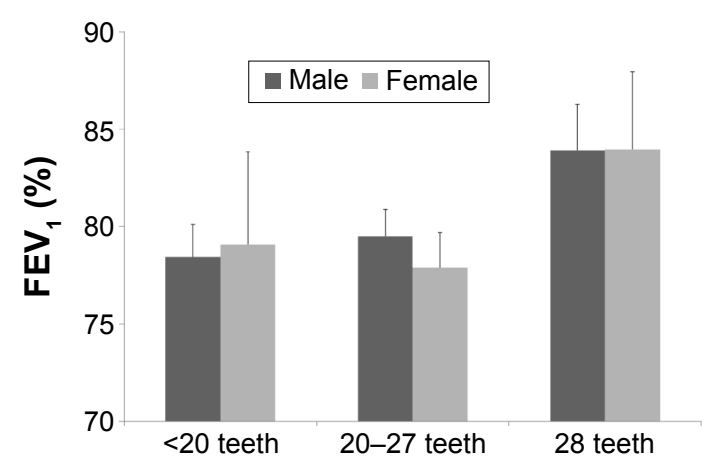

Figure 2 FEV, with subgroups of the number of natural teeth in patients with airflow obstruction.

Notes: Although there were no significant differences in both sexes, FEV , showed a decreasing trend in the groups with 20-27 teeth and $<20$ teeth compared with the 28-teeth group after adjustment for confounding factors (age, smoking, drinking, exercise, income, education, and BMI).

Abbreviations: $\mathrm{FEV}_{\mathrm{I}}$, forced expiratory volume in I second; BMI, body mass index. major periodontal factor for predicting $\mathrm{COPD}(\mathrm{OR}=9.01$, 95\% CI $=3.98-20.4)$.

Although oral conditions likely work in concert with other factors and the underlying biological mechanisms still remain obscure, ${ }^{16}$ several mechanisms were suggested in these various studies to explain the association of COPD with poor oral health. Aspiration of oral pathogens into the lower respiratory tract was one of these hypotheses. ${ }^{31}$ Periodontal disease-associated enzymes in saliva may also change respiratory environmental conditions, permitting mucosal colonization and infection by respiratory pathogens. ${ }^{15,28,31}$ In addition, the association between poor oral health and respiratory disease was suggested to be mediated through systemic inflammatory responses. ${ }^{9,32}$ Inflammatory cytokines, such as interleukin (IL)-6 or IL-8, from periodontal tissues may alter the respiratory epithelium to promote infection by respiratory pathogens ${ }^{16}$ From other studies, tooth loss or less frequently brushing teeth was associated with an increased serum level of C-reactive protein. ${ }^{33-35}$ Levels of inflammatory biomarkers such as C-reactive protein and IL-6 also have been reported to be elevated in COPD. ${ }^{11}$

In this study, we used the number of natural teeth as a measure of oral health. Rather than actually investigating oral hygiene or the presence of periodontitis, the number of natural teeth is a rapid and easy index for both clinicians and patients. Changes in oral health status due to caries and periodontitis lead to a reduced number of teeth. ${ }^{36}$ Additionally, Kressin et $\mathrm{al}^{37}$ showed that many oral health behaviors, including brushing, flossing, and prophylactic dental visits, can help retain a greater number of natural teeth. ${ }^{37}$ In our results, only number of natural teeth was significantly related with airflow obstruction, rather than periodontitis, number of tooth brushings, or the use of secondary oral products. We believe that the cumulative effects of diverse oral health factors are reflected in the number of teeth. We used pairs of natural teeth as a marker to reflect dental function, such 
as biting and chewing. In several other studies, functional units, defined as any opposing natural or prosthetic tooth pair, were used to account for the functional arrangement of the teeth. ${ }^{38-40}$ From our results, both number and pairs of natural teeth showed an almost identical correlation with airflow obstruction. Males who had low numbers or pairs of teeth showed a stronger relationship with airflow obstruction.

In both sexes, patients with airflow obstruction tended to have the following characteristics: older in age, lower BMI, lower income, and less education. Subjects with restrictive lung patterns showed higher BMI, WC, and presence of DM and metabolic syndrome in both males and females. However, only male subjects with airflow obstruction comprised a significantly greater number of smokers, partook in less exercise, and had a lower total energy intake. This difference may be because Korean females in the general population traditionally have a low smoking rate, exercise, and total energy intake. Contrary to our expectations, levels of vitamin $\mathrm{D}$ were significantly higher in males with airflow obstruction. Several studies have reported a high prevalence of vitamin D deficiency in COPD patients. ${ }^{41-44}$ This may be explained by the nature of the study population. All study groups showed vitamin D deficiency $(<20 \mathrm{ng} / \mathrm{mL})$ regardless of lung function, and the group with airflow obstruction consisted mostly of mild cases.

After adjustments, the number or pairs of teeth were significantly related to airflow obstruction only in males. In females, other confounding factors, such as hormonal effects and the frequency of dental checkups, which were not considered in this study, may have influenced the results. Additionally, the relationship between the number of natural teeth and airflow obstruction in females according to the severity of airflow obstruction should be compared in further studies.

Our study had several limitations. First, postbronchodilator spirometry was not performed in KNHANES. Using the results of prebronchodilator spirometry for diagnosing COPD may overestimate the number of COPD patients. Therefore, we defined the obstructive pattern of spirometry as "airflow obstruction" instead of COPD. Second, the study was of a cross-sectional design and so no cause-effect relationship could be determined. Third, this study involved healthy patients with mild airflow obstruction rather than those with severe airflow obstruction. KNHANES data were obtained from subjects participating in a nationwide survey; individuals who were admitted to hospitals or nursing homes were not included. Within these limitations, the KNHANES data represent the public health of the total population of Korea and is of considerable value in many respects. ${ }^{45}$

Despite these limitations, there are several strengths in our study. First, the number of teeth and oral condition was objectively assessed by dentist with same criteria. In addition, several physical examinations and important confounding variables were also precisely recorded by trained examiners. Second, our study included a large number of participants who represent the Korean population. Third, we used the number of natural teeth, which is a convenient index and reflects the cumulative effects of oral health.

\section{Conclusion}

Loss of natural teeth was significantly associated with the presence of airflow obstruction in Korean males. Based on our results, the number of natural teeth could be one of the available indices for obstructive lung diseases, including COPD. Further investigations are needed to clarify the relationship and mechanism between the oral health and obstructive lung diseases.

\section{Acknowledgment}

This study was funded by a research grant from the Institute of Clinical Medicine Research, Yeouido St Mary's Hospital, The Catholic University of Korea.

\section{Disclosure}

The authors report no conflicts of interest in this work.

\section{References}

1. Vestbo J, Hurd SS, Agusti AG, et al. Global strategy for the diagnosis, management, and prevention of chronic obstructive pulmonary disease: GOLD executive summary. Am J Respir Crit Care Med. 2013 187(4):347-365.

2. May SM, Li JT. Burden of chronic obstructive pulmonary disease: healthcare costs and beyond. Allergy Asthma Proc. 2015;36(1):4-10.

3. Pauwels RA, Rabe KF. Burden and clinical features of chronic obstructive pulmonary disease (COPD). Lancet. 2004;364(9434):613-620.

4. Murray CJ, Lopez AD. Alternative projections of mortality and disability by cause 1990-2020: Global Burden of Disease Study. Lancet. 1997; 349(9064):1498-1504.

5. Tsai TY, Livneh H, Lu MC, Tsai PY, Chen PC, Sung FC. Increased risk and related factors of depression among patients with COPD: a population-based cohort study. BMC Public Health. 2013;13:976.

6. Sullivan SD, Ramsey SD, Lee TA. The economic burden of COPD. Chest. 2000;117(2 Suppl):5s-9s.

7. Chapman KR, Mannino DM, Soriano JB, et al. Epidemiology and costs of chronic obstructive pulmonary disease. Eur Respir J. 2006;27(1) 188-207.

8. Celli BR, MacNee W. Standards for the diagnosis and treatment of patients with COPD: a summary of the ATS/ERS position paper. Eur Respir J. 2004;23(6):932-946. 
9. Barnes PJ, Celli BR. Systemic manifestations and comorbidities of COPD. Eur Respir J. 2009;33(5):1165-1185.

10. Rabe KF, Hurd S, Anzueto A, et al. Global strategy for the diagnosis, management, and prevention of chronic obstructive pulmonary disease: GOLD executive summary. Am J Respir Crit Care Med. 2007;176(6): $532-555$.

11. Barros SP, Suruki R, Loewy ZG, Beck JD, Offenbacher S. A cohort study of the impact of tooth loss and periodontal disease on respiratory events among COPD subjects: modulatory role of systemic biomarkers of inflammation. PLoS One. 2013;8(8):e68592.

12. Scannapieco FA, Papandonatos GD, Dunford RG. Associations between oral conditions and respiratory disease in a national sample survey population. Ann Periodontol. 1998;3(1):251-256.

13. Azarpazhooh A, Leake JL. Systematic review of the association between respiratory diseases and oral health. J Periodontol. 2006;77(9): $1465-1482$.

14. Zhou X, Wang Z, Song Y, Zhang J, Wang C. Periodontal health and quality of life in patients with chronic obstructive pulmonary disease. Respir Med. 2011;105(1):67-73.

15. Scannapieco FA, Mylotte JM. Relationships between periodontal disease and bacterial pneumonia. J Periodontol. 1996;67(10 Suppl): 1114-1122.

16. Scannapieco FA, Ho AW. Potential associations between chronic respiratory disease and periodontal disease: analysis of National Health and Nutrition Examination Survey III. J Periodontol. 2001;72(1):50-56.

17. Öztekin G, Baser U, Kucukcoskun M, et al. The association between periodontal disease and chronic obstructive pulmonary disease: a case control study. COPD. 2014;11(4):424-430.

18. Korea Centers for Disease Control and Prevention (2015). Korea National Health and Nutrition Examination Survey (KNHANES), 2012. Available from: https://knhanes.cdc.go.kr/knhanes/index.do. Accessed March 27, 2015.

19. Grundy SM, Cleeman JI, Daniels SR, et al. Diagnosis and management of the metabolic syndrome: an American Heart Association/National Heart, Lung, and Blood Institute Scientific Statement. Circulation. 2005; 112(17):2735-2752.

20. Lehouck A, Boonen S, Decramer M, Janssens W. COPD, bone metabolism, and osteoporosis. Chest. 2011;139(3):648-657.

21. Jimenez M, Giovannucci E, Krall Kaye E, Joshipura KJ, Dietrich T. Predicted vitamin D status and incidence of tooth loss and periodontitis. Public Health Nutr. 2014;17(4):844-852.

22. Ranu H, Wilde M, Madden B. Pulmonary function tests. Ulster Med J. 2011;80(2):84-90

23. Park HJ, Leem AY, Lee SH, et al. Comorbidities in obstructive lung disease in Korea: data from the fourth and fifth Korean National Health and Nutrition Examination Survey. Int J Chron Obstruct Pulmon Dis. 2015; 10:1571-1582.

24. Chung JH, Hwang HJ, Han CH, Son BS, Kim do H, Park MS. Association between sarcopenia and metabolic syndrome in chronic obstructive pulmonary disease: the Korea National Health and Nutrition Examination Survey (KNHANES) from 2008 to 2011. COPD. 2015; 12(1):82-89.

25. Lee JB, Yi HY, Bae KH. The association between periodontitis and dyslipidemia based on the Fourth Korea National Health and Nutrition Examination Survey. J Clin Periodontol. 2013;40(5):437-442.

26. Kingman A, Susin C, Albandar JM. Effect of partial recording protocols on severity estimates of periodontal disease. J Clin Periodontol. 2008;35(8):659-667.

27. Si Y, Fan H, Song Y, Zhou X, Zhang J, Wang Z. Association between periodontitis and chronic obstructive pulmonary disease in a Chinese population. J Periodontol. 2012;83(10):1288-1296.
28. Hayes C, Sparrow D, Cohen M, Vokonas PS, Garcia RI. The association between alveolar bone loss and pulmonary function: the VA Dental Longitudinal Study. Ann Periodontol. 1998;3(1):257-261.

29. Deo V, Bhongade ML, Ansari S, Chavan RS. Periodontitis as a potential risk factor for chronic obstructive pulmonary disease: a retrospective study. Indian J Dent Res. 2009;20(4):466-470.

30. Leuckfeld I, Obregon-Whittle MV, Lund MB, Geiran O, Bjortuft O, Olsen I. Severe chronic obstructive pulmonary disease: association with marginal bone loss in periodontitis. Respir Med. 2008;102(4): 488-494.

31. Vadiraj S, Nayak R, Choudhary GK, Kudyar N, Spoorthi BR. Periodontal pathogens and respiratory diseases-evaluating their potential association: a clinical and microbiological study. J Contemp Dent Pract. 2013;14(4):610-615.

32. Montebugnoli L, Servidio D, Miaton RA, Prati C, Tricoci P, Melloni C. Poor oral health is associated with coronary heart disease and elevated systemic inflammatory and haemostatic factors. J Clin Periodontol. 2004; 31(1):25-29.

33. Kobayashi Y, Niu K, Guan L, et al. Oral health behavior and metabolic syndrome and its components in adults. J Dent Res. 2012; 91(5):479-484.

34. Holmlund A, Hulthe J, Lind L. Tooth loss is related to the presence of metabolic syndrome and inflammation in elderly subjects: a prospective study of the vasculature in Uppsala seniors (PIVUS). Oral Health Prev Dent. 2007;5(2):125-130.

35. Janket SJ, Baird AE, Jones JA, et al. Number of teeth, C-reactive protein, fibrinogen and cardiovascular mortality: a 15-year follow-up study in a Finnish cohort. J Clin Periodontol. 2014;41(2):131-140.

36. Witter DJ, van Palenstein Helderman WH, Creugers NH, Kayser AF. The shortened dental arch concept and its implications for oral health care. Community Dent Oral Epidemiol. 1999;27(4):249-258.

37. Kressin NR, Boehmer U, Nunn ME, Spiro A 3rd. Increased preventive practices lead to greater tooth retention. J Dent Res. 2003;82(3): 223-227.

38. Hildebrandt GH, Dominguez BL, Schork MA, Loesche WJ. Functional units, chewing, swallowing, and food avoidance among the elderly. J Prosthet Dent. 1997;77(6):588-595.

39. Hsu KJ, Yen YY, Lan SJ, Wu YM, Lee HE. Impact of oral health behaviours and oral habits on the number of remaining teeth in older Taiwanese dentate adults. Oral Health Prev Dent. 2013;11(2):121-130.

40. Naka O, Anastassiadou V, Pissiotis A. Association between functional tooth units and chewing ability in older adults: a systematic review. Gerodontology. 2014;31(3):166-177.

41. Jung JY, Kim YS, Kim SK, et al. Relationship of vitamin D status with lung function and exercise capacity in COPD. Respirology. 2015; 5:782-789.

42. Persson LJ, Aanerud M, Hiemstra PS, et al. Vitamin D, vitamin D binding protein, and longitudinal outcomes in COPD. PLoS One. 2015; 10(3): 0121622.

43. Martineau AR, James WY, Hooper RL, et al. Vitamin D3 supplementation in patients with chronic obstructive pulmonary disease (ViDiCO): a multicentre, double-blind, randomised controlled trial. Lancet Respir Med. 2015;3(2):120-130.

44. Skaaby T, Husemoen LL, Thuesen BH, et al. Vitamin D status and chronic obstructive pulmonary disease: a prospective general population study. PLoS One. 2014;9(3):e90654.

45. Kim Y. The Korea National Health and Nutrition Examination Survey (KNHANES): current status and challenges. Epidemiol Health. 2014; 36:e2014002. 


\section{Supplementary material}

Table SI Standard of monthly household income* (US dollar)

\begin{tabular}{|c|c|c|c|c|c|c|}
\hline \multirow{2}{*}{$\begin{array}{l}\text { Age } \\
\text { (years) }\end{array}$} & \multicolumn{3}{|l|}{ Male } & \multicolumn{3}{|l|}{ Female } \\
\hline & Quartile I & Quartile 2 & Quartile 3 & Quartile I & Quartile 2 & Quartile 3 \\
\hline $40-44$ & $\mathrm{I}, 262$ & $\mathrm{I}, 730$ & 2,523 & $\mathrm{I}, 083$ & $\mathrm{I}, 709$ & 2,549 \\
\hline $45-49$ & 1,249 & 1,730 & 2,497 & 1,165 & I,848 & 2,549 \\
\hline $50-54$ & $\mathrm{I}, 08 \mathrm{I}$ & $\mathrm{I}, 74 \mathrm{I}$ & 2,882 & $\mathrm{I}, 040$ & $\mathrm{I}, 730$ & 2,610 \\
\hline $55-59$ & 999 & $\mathrm{I}, 730$ & 2,572 & 918 & $\mathrm{I}, 529$ & 2,247 \\
\hline $60-64$ & 918 & $|, 5| 3$ & 2,289 & 566 & 1,199 & 1,859 \\
\hline $65-69$ & 563 & 999 & 1,648 & 459 & 816 & $\mathrm{I}, 498$ \\
\hline $70-74$ & 422 & 805 & 1,348 & 300 & 508 & 967 \\
\hline $75+$ & 275 & 483 & 883 & 242 & 425 & 979 \\
\hline
\end{tabular}

Notes: Data from the Korean National Health and Nutrition Examination Survey (KNHANES), $2012 .{ }^{18}$ *The Korean National Health and Nutrition Examination Survey provides the value of monthly income after standardization according to the number of family members.

\section{Publish your work in this journal}

The International Journal of COPD is an international, peer-reviewed journal of therapeutics and pharmacology focusing on concise rapid reporting of clinical studies and reviews in COPD. Special focus is given to the pathophysiological processes underlying the disease, intervention programs, patient focused education, and self management protocols.

\section{Dovepress}

This journal is indexed on PubMed Central, MedLine and CAS. The manuscript management system is completely online and includes a very quick and fair peer-review system, which is all easy to use. Visit http://www.dovepress.com/testimonials.php to read real quotes from published authors. 